\title{
THE NON-LINEAR ENSKOG-BOLTZMANN EQUATION
}

\author{
H. Van BEIJEREN \\ Instituut voor Theoretische Fysica, Katholieke Universiteit, \\ Nijmegen, The Netherlands \\ and \\ M.H. ERNST \\ Instituut voor Theoretische Fysica, Rijksuniversiteit, \\ Utrecht, The Netherlands
}

Received 30 January 1973

\begin{abstract}
Some implications of a modified form of the non-linear Enskog equation for a hard sphere fluid are investigated. It leads to different Navier-Stokes equations in a multicomponent mixture, and its linearized form describes the space and time dependence fluctuations.
\end{abstract}

Starting from the Liouville equation for the nonequilibrium $N$-particle distribution function, we have derived a modified form of the non-linear Enskog equation. At the initial time, $t=0$, we have taken into account all hard sphere overlap exclusions, but all other position and velocity correlations are neglected. Our modified Enskog equation becomes exact, under these conditions, for times much shorter that the mean free time between collisions. It, therefore, describes on this time scale the single particle distribution function $f(x, t)$ for arbitrary spatial variations. The derivation will be given in a future publication. Here we will point out some interesting implications of this eqaution, which reads

$\frac{\partial}{\partial t} f_{1}+v_{1} \cdot \nabla f_{1}=\int \mathrm{d} x_{2} T_{12} \bar{\chi}\left(r_{1} r_{2}\right) f_{1} f_{2}$,

where $f_{i}=f\left(x_{i}, t\right)$ and $x_{i}=\left(r_{i}, v_{i}\right)$ stands for the position and velocity of particle $i(i=1,2) ; T_{12}$ is the binary collision operator, and $\bar{\chi}\left(\boldsymbol{r}_{1} \boldsymbol{r}_{2}\right)$ the local pair distribution function for $r_{12} \geqslant$ the diameter of the spheres $\sigma$. The definitions are

$$
\begin{aligned}
& T_{12}=\sigma^{2} \int^{\prime} \mathrm{d} \hat{\boldsymbol{\sigma}}\left(v_{12} \cdot \hat{\boldsymbol{\sigma}}\right)\left\{\delta\left(v_{12}-\boldsymbol{\sigma}\right) R_{\boldsymbol{\sigma}}-\delta\left(\boldsymbol{r}_{12}+\boldsymbol{\sigma}\right)\right\} \\
& \bar{\chi}\left(\boldsymbol{r}_{1} \boldsymbol{r}_{2}\right)=1+\int \mathrm{d} \boldsymbol{r}_{3} n\left(\boldsymbol{r}_{3}\right) V(12 \mid 3) \\
& \quad+\frac{1}{2 !} \int \mathrm{d} r_{3} \mathrm{~d} \boldsymbol{r}_{4} n\left(r_{3}\right) n\left(r_{4}\right) V(12 \mid 34)+\ldots
\end{aligned}
$$

The prime on the integral indicates that $v_{12} \cdot \hat{\boldsymbol{\sigma}}>0$, where $v_{12}=v_{1}-v_{2}$, and $\hat{\sigma}=\sigma / \sigma$ is a unit vector. The operator $R_{\boldsymbol{\sigma}}$ transforms the precollisional velocities $v_{1}$ and $v_{2}$ into the postcollisional velocities $v_{1}^{*}$ and $v_{2}^{*}$ resp. eq. (3) has the form of the pair distribution function for a fluid in a non-uniform equilibrium state, where in our case the local density $n(r, t)=\int \mathrm{d} v f(x, t)$. The Husimifunctions $V(12 \mid 3 \ldots)$ are the same as enter in the density expansion of the equilibrium pair distribution function [1]. In the usual Enskog equation [2] $\bar{\chi}\left(r_{1} r_{2}\right)$ is replaced by the local pair distribution function $\chi\left(r_{12} \mid n(R)\right)$, localized at the fixed point $\boldsymbol{R}=$ $\frac{1}{2}\left(r_{1}+r_{2}\right)$. This means that all local densities $n\left(r_{i}\right)$ in eq. (3) are replaced by $n(R)$. Intuitively $\bar{\chi}\left(r_{1} r_{2}\right)$ in eq. (1) seems also appealing, since it takes in to account the spatial correlations between two spheres in a nonuniform local equilibrium state, while in the usual Enskog equation non-uniformities are only taken into account to a certain extent. To see this we expand the local densities in eq. (3) around some fixed point $R$. By choosing $R=\frac{1}{2}\left(r_{1}+r_{2}\right)$ the term linear in the gradient vanishes and we have

$\bar{\chi}\left(r_{1} r_{2}\right)=\chi\left(r_{12} \ln (R)\right)+O\left(\nabla^{2}\right)$.

Hence, up to terms of $O\left(\nabla^{2}\right)$ eq. (1) is identical with the usual Enskog equation. If one would use eq. (1) for the calculation of transport coefficients and for the derivation of hydrodynamic equations (which is strictly speaking outside the time regime for which 
eq. (1) has been derived) it gives the usual Enskog results at the Navier-Stokes level. However, our equation may also be used to derive Burnett and higher order hydrodynamic equations, while the usual Enskog equation can no longer be used [2]. For multicomponent mixtures, extensions of the Enskog collision term have been given $[2,3]$ in which the local pair distribution function for two particles of different species in contact has been localized either at the point of contact, or at the mid point between the centres of the two colliding spheres. The two choices give different Navier-Stokes equations. Neither of these choices is consistent with eq. (1) in the case of mixtures for the following reason. If one expands the local pair distribution function for two particles belonging to different species around some point $\boldsymbol{R}$, it is impossible to choose the point $R$ in such a way that the terms linear in the gradients vanish for all values of the partial densities.

Consequently extension of eq. (1) to multicomponent mixtures leads to an Enskog equation which differs already at first order in the gradients from the equations in refs. [2,3] and, therefore, yields different Navier-Stokes equations. In the way the theories are presented, the differences manifest themselves mainly in the form of the diffusion forces. For instance, the diffusion forces at uniform temperature in refs. [2,3] coincide only with the gradients of the chemical potentials to lowest and first order in the density, while in our case the diffusion forces are found directly, as the gradients of the chemical potentials to all orders in the density, as it should be according to irreversible thermodynamics. The diffusion coefficients however are identical in all cases.

An other interesting consequence of eq. (1) is its linearized form. We have shown that linearization of eq. (1) around total equilibrium reduces to the following equation for the Fourier-transform, $\widetilde{\psi}(k, v, t)$, of the quantity $\phi^{-1}(v)\{f(x, t)-\phi(v)\}$, where $\phi(v)$ is the Maxwell-Boltzmann distribution function multiplied by the equilibrium density $n$; and $\boldsymbol{k}$ the Fourier variable:

$$
\begin{aligned}
& \frac{\partial}{\partial t} \widetilde{\psi}+\mathrm{i} k \cdot v \widetilde{\psi}=\Lambda_{\mathrm{k}} \widetilde{\psi}+\mathrm{i} k \cdot \mathrm{v}\left(\widetilde{C}_{\mathrm{k}}-\chi \widetilde{f}_{\mathrm{k}}\right) \tilde{n}_{\mathrm{k}} \\
& \Lambda_{\mathrm{k}} \widetilde{\psi}\left(\mathbf{v}_{1}\right)=n \chi \sigma^{2} \int \mathrm{a} \mathbf{v}_{2} \phi\left(v_{2}\right) \int \mathrm{d} \hat{\boldsymbol{\sigma}}\left(\mathbf{v}_{12} \cdot \hat{\boldsymbol{\sigma}}\right) \\
& \quad \times\left\{\widetilde{\psi}\left(\mathbf{v}_{1}^{*}\right)+\widetilde{\psi}\left(\mathbf{v}_{2}^{*}\right) \exp (-\mathrm{i} k \cdot \boldsymbol{\sigma})\right. \\
& \left.-\widetilde{\psi}\left(v_{1}\right)-\widetilde{\psi}\left(\mathbf{v}_{2}\right) \exp (\mathrm{i} k \cdot \sigma)\right\} .
\end{aligned}
$$

Here $\chi=\chi(\sigma \mid n) ; \tilde{n}_{\mathrm{k}}, \widetilde{C}_{\mathrm{k}}, \tilde{f}_{\mathrm{k}}$ are the Fourier-transforms resp. of $[n(r, t)-n]$, of the Ornstein-Zernike direct correlation function, and of the Mayer function $f(r)[f(r)=-1$ for $r<\sigma$, and vanishes elsewhere $]$. The second term on the right-hand side of eq. (5) results from linearization of $\bar{\chi}\left(r_{1} r_{2}\right)$. Eq. (5) was recently derived by means of many body methods by Mazenko et al. [4], and by Konijnendijk and Van Leeuwen [5], for a description of the short time behaviour of space and time dependent fluctuations in a hard sphere fluid.

A detailed account of the calculations will be published elsewhere.

We thank Professor E.G.D. Cohen for some helpful comments.

\section{References}

[1] Studies in Statistical Mechanics I, Ed. J. De Boer and G. Uhlenbeck, (North Holland, Amsterdam, 1962) p.163.

[2] S. Chapman and T.G. Cowling, The mathematical theory of non-uniform gases (Cambridge, University Press, 1960) p. 292.

[3] L.S. Carcia-Colin, L. Barajas and E. Pina, Phys. Lett. 37A (1971) 395 and references cited therein.

[4] G.F. Mazenko, T.Y.C. Wei and S. Yip, Phys. Rev. A6 (1972) 1981.

[5] H.H.U. Konijnendijk and J.M.J. Van Leeuwen, Physica, to be published. 